\title{
A Novel Algorithm for MIMO Signal Classification Using Higher-Order Cumulants
}

\author{
Michael S. Mühlhaus*, Mengüc Öner ${ }^{\dagger}$, Octavia A. Dobre ${ }^{\ddagger}$, Holger U. Jäkel ${ }^{*}$, and Friedrich K. Jondral* \\ * Karlsruhe Institute of Technology, Germany, Email: \{michael.muehlhaus, holger.jaekel, friedrich.jondral\}@kit.edu \\ ${ }^{\dagger}$ Isik University, Sile, Turkey, Email: oner@isikun.edu.tr \\ $\ddagger$ Memorial University, St. John’s, Canada, Email: odobre@mun.ca
}

\begin{abstract}
Automatic modulation classification (AMC) of unknown communications signals is employed in both commercial and military applications, such as cognitive radio, spectrum surveillance, and electronic warfare. Most of the AMC methods proposed in the literature are developed for systems with a single transmit antenna. In this paper, an AMC algorithm for multipleinput multiple-output (MIMO) signals is proposed, which is based on higher-order cumulants. The use of cumulants with different orders, as well as their combinations as feature vectors are investigated. The ideal case of a priori knowledge of the channel state information (CSI) is considered, along with a setting of practical relevance, where the channel matrix is blindly estimated through independent component analysis. The performance of the proposed algorithm with different features is evaluated through simulations and compared with that of the average likelihood ratio test (ALRT).
\end{abstract}

Index Terms-Automatic modulation classification, multipleinput multiple-output, independent component analysis, blind source separation, higher-order cumulants.

\section{INTRODUCTION}

Automatic modulation classification (AMC) algorithms are employed to determine the modulation type of unknown communications signals, with applications to cognitive radio, spectrum surveillance, and signals intelligence.

Two different approaches to the AMC problem can be observed in the literature for single-input single-output (SISO) systems, i.e. the likelihood- and feature-based algorithms [1][5], respectively. The former is optimal in the Bayesian sense, but requires a priori knowledge of the parameters of the transmitted signal, and displays a high computational complexity. The latter extracts features from the received signal to discriminate the candidate modulation types, and can be regarded as sub-optimal but usually requires little $a$ priori information. The computational complexity is lower than that of the likelihoodbased approach [6]. Choqueuse et al. extended the average likelihood ratio test (ALRT) to MIMO spatial multiplexing systems [7]. In this contribution, we propose a feature-based AMC algorithm for MIMO systems that employs higher-order cumulants of the received signal as discriminating features.

The remainder of the paper is organized as follows. In Section II, the MIMO system model is described. The proposed algorithm is introduced in Section III. In Section IV, the performance of the algorithm using different feature vectors

This work was partially supported by the TUBITAK (The Scientific and Technological Research Council of Turkey) grant no: $112 \mathrm{E} 020$ is investigated through simulations, with and without channel state information (CSI). These results are compared with ARLT. The paper concludes with Section V.

\section{SYSTEM MODEL}

A spatial multiplexing MIMO system with $N_{t}$ transmit and $N_{r} \geq N_{t}$ receive antennas multiplexes the modulated transmit symbol stream to $N_{t}$ parallel independent symbol streams, which are directly fed to the transmit antennas; hence, the transmitted symbols are independent in time and space. The received signal $\mathbf{r}[k]=\left[r_{1}[k], \ldots, r_{N_{r}}[k]\right]^{T}$ at time instant $k$ is given by

$$
\mathbf{r}[k]=\mathbf{H s}[k]+\mathbf{w}[k],
$$

where $\mathbf{s}[k]=\left[s_{1}[k], \ldots, s_{N_{t}}[k]\right]^{T}$ is a vector containing the transmitted symbols from all antennas, and $\mathbf{w}[k]$ is a white noise vector whose elements are zero-mean complex Gaussian distributed random variables with variance $\sigma_{w}^{2}$. The MIMO channel is characterized by the channel matrix

$$
\mathbf{H}=\left[\begin{array}{ccc}
h_{1,1} & \cdots & h_{1, N_{t}} \\
\vdots & \ddots & \vdots \\
h_{N_{r}, 1} & \cdots & h_{N_{r}, N_{t}}
\end{array}\right],
$$

where $h_{m, l}, m=1, \ldots, N_{r}, l=1, \ldots, N_{t}$, is the channel coefficient between the $m$-th receive and $l$-th transmit antenna. We consider a Rayleigh block fading channel. The average signal-to-noise ratio (SNR) is defined as the total received mean signal power $N_{t} N_{r} \sigma_{s}^{2}$ divided by the total noise power $N_{r} \sigma_{w}^{2}$. Assuming, w.l.o.g. that the variance $\sigma_{s}^{2}$ of the transmitted symbols is normalized to one the SNR is $\frac{N_{t}}{\sigma_{w}^{2}}$.

\section{Proposed Feature-Based Classification AlgorithM}

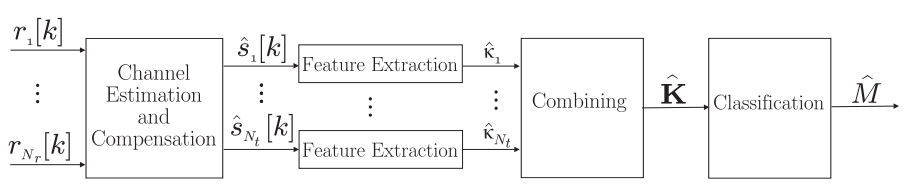

Fig. 1. The proposed AMC architecture.

In this work, we propose a feature-based AMC approach, that uses higher-order cumulants as discriminating features. The proposed AMC architecture is depicted in Fig. 1 [8]. The signal of interest is received using a MIMO receiver with $N_{r}$ 
antennas. Subsequently, the channel is compensated in order to recover the $N_{t}$ parallel noisy independent symbol streams, $\hat{s}_{m}[k], m=1, \ldots, N_{t}$. Note that each stream corresponds to the signal from one transmit antenna. Then, modulation typespecific features are estimated from each of the $N_{t}$ estimated independent symbol streams individually, which finally are combined to be used for classification.

With perfect CSI, the channel compensation can be carried out using zero forcing (ZF). The received signal vector $\mathbf{r}[k]$ is multiplied by the pseudo-inverse of the channel matrix $\mathbf{H}^{+}:=$ $\left(\mathbf{H}^{\mathrm{H}} \mathbf{H}\right)^{-1} \mathbf{H}^{\mathrm{H}}$, where $(\cdot)^{\mathrm{H}}$ denotes the Hermitian operation.

If the channel matrix is not known, which is a much more realistic assumption for scenarios where AMC systems are used, the transmit symbols are estimated by the well known joint approximate diagonalization of eigen-matrices (JADE) algorithm proposed in [9], using solely the received signal samples at each receive antenna, without requiring any a priori information, except for the number of transmit antennas, $N_{t}$. Note that JADE is able to separate the independent signal streams only up to a real-valued multiplicative factor and a phase rotation. Thus, the estimated transmit signals must be normalized as follows

$$
\bar{s}_{m}[k]=\frac{\hat{s}_{m}[k] \sqrt{1+P_{m}}}{\sigma_{\hat{s}_{m}}},
$$

where $P_{m}$ is the noise power of the $m$-th estimated symbol stream. Furthermore, the discriminating features used for modulation type classification should be chosen such that they are robust to unknown phase offsets. After the equalization, the filtered noise $\tilde{\mathbf{w}}[k]$ is clearly no longer white; hence its components are correlated and have non-equal powers.

For classification feature vectors containing higher-order cumulants are used. The general expression of a cumulant of order $u, v$-times conjugated, for a complex random variable $s$ is given as [10]

$$
\kappa_{s}^{u, v}=\sum_{P_{u}}\left[k(p) \prod_{j=1}^{p} \mathrm{E}\left\{s^{u_{j}-v_{j}} s^{* v_{j}}\right\}\right],
$$

where $P_{u}$ is the set of the partitions of the elements $\{1,2, \ldots, u\}$. A partition $\rho$ consists of $p$ sets $\nu_{j}: \rho=\left\{\nu_{j}\right\}_{j=1}^{p}$, $u_{j}$ is the size of the set $\nu_{j}, v_{j}$ is the number of conjugated terms, $k(p)=(-1)^{p-1}(p-1)$ ! and $\mathrm{E}\{\cdot\}$ denotes expectation. For further details, the reader is referred to [10].

Theoretical values for cumulants of diverse orders for different linear modulation types are provided in following table:

\begin{tabular}{|c|c|c|c|c|}
\hline & BPSK & QPSK & 8 -PSK & 16-QAM \\
\hline$\kappa_{S}^{2,0}$ & 1 & 0 & 0 & 0 \\
$\kappa_{S}^{2,1}$ & 1 & 1 & 1 & 1 \\
\hline$\kappa_{S}^{4,0}$ & -2 & 1 & 0 & -0.68 \\
$\kappa_{S, 1}^{4,1}$ & -2 & 0 & 0 & 0 \\
$\kappa_{S}^{4,2}$ & -2 & -1 & -1 & -0.68 \\
\hline$\kappa_{S}^{6,0}$ & 16 & 0 & 0 & 0 \\
$\kappa_{S}^{6,1}$ & 16 & -4 & 0 & 2.08 \\
$\kappa_{\delta, 2}^{6,2}$ & 16 & 0 & 0 & 0 \\
$\kappa_{S}^{6,3}$ & 16 & 4 & 4 & 2.08 \\
\hline$\kappa_{\mathcal{8}}^{8,0}$ & -272 & -34 & 1 & -13.981 \\
$\kappa_{\mathcal{S}}^{8,1}$ & -272 & 0 & 0 & 0 \\
$\kappa_{S}^{8,2}$ & -272 & 34 & 0 & -13.981 \\
$\kappa_{\mathcal{S}, 3}^{8,3}$ & -272 & 0 & 0 & 0 \\
$\kappa_{S}^{8,4}$ & -272 & -34 & -33 & -13.981 \\
\hline
\end{tabular}

Only cumulants of even order are non-zero for linearly modulated signals, due to the symmetry of signal constellation points. It can be seen that some cumulants have identical values for different modulation schemes, and hence cannot be used for their discrimination. Furthermore, the cumulant value also depend on the phase offsets, which may be present in the recovered symbol streams. Thus, we choose to use the magnitudes of the estimated cumulants for classification, in order to make the features invariant to phase offsets.

Following the feature extraction stage, the feature vectors estimated from different symbol streams are combined prior to the classification stage. We choose to employ selection combining ${ }^{1}$ i.e., using the feature estimate from the signal branch with the highest SNR.

Finally, the decision on a modulation scheme $M_{i}$ is made in the classification stage by choosing the modulation type that minimizes the Euclidean distance between the combined feature vector estimate $\hat{\mathbf{K}}$ and the theoretical feature vector, $\mathbf{K}_{M_{q}}$.

\section{Simulation Results}

In this section, simulations are used to evaluate the performance of the proposed AMC algorithm with various cumulantbased features. The average probability of correct classification $P_{C C}$ is employed as a performance measure.

The average likelihood ratio test (ALRT), that has been derived in [7] under the assumption of perfect CSI, is used a performance benchmark. It is optimal in the Bayesian sense, given that the channel matrix and the noise variance are known, but has a complexity, which severely limits the applicability.

Unless otherwise mentioned, the following parameters are used in simulations. The set of modulations is $\mathcal{M}=$ \{BPSK, QPSK, 8 - PSK, $16-$ QAM , the number of transmit antennas is $N_{t}=2$, the number of receive antennas is $N_{r}=4$, and the observation length is $T=1000$. For each SNR value, 2000 iterations were performed.

Fig. 2 displays the performance of the proposed algorithm for a single cumulant-based feature of various orders and ZF equalization, as well as the performance of the ALRT.

The increase in the order of the employed cumulant feature leads to the following opposite effects. On one hand, the distance between the theoretical values of the cumulant features for different modulation schemes increases, improving the ability of the algorithm to discriminate between the modulation types. On the other hand, the variance of the cumulant estimates increases, which degrades the classification performance. The performance of the cumulant-based features $\left|\kappa_{s}^{8,2}\right|$ and $\left|\kappa_{s}^{6,1}\right|$ is similar and about $0.5 \mathrm{~dB}$ better than $\left|\kappa_{s}^{4,0}\right|$, while the feature $\left|\kappa_{s}^{8,0}\right|$ has the worst classification rate. When compared with ALRT, an SNR increase of about 5.5 $\mathrm{dB}$ is required to achieve $P_{C C}=0.9$ with the $\left|\kappa_{s}^{8,2}\right|$-based algorithm.

\footnotetext{
${ }^{1}$ A detailed investigation of combining schemes for the feature vectors will be provided in future work and is therefore out of the scope of this paper.
} 


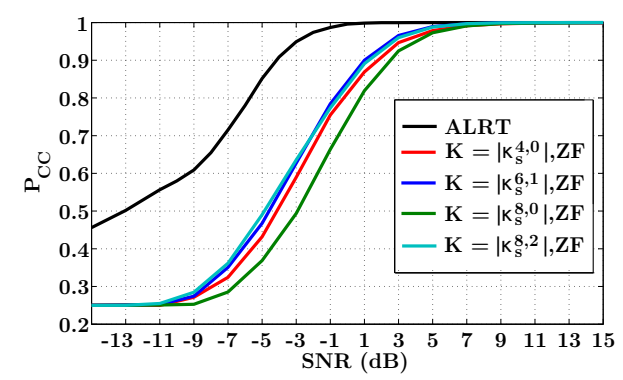

Fig. 2. Performance of the proposed algorithm with different order cumulants and perfect CSI using ZF, in comparison to ALRT.

The combination of different features in vectors leads to a higher degree of freedom in designing the AMC system. By using this approach, it becomes possible to employ features that, by themselves, cannot classify all the modulation types in $\mathcal{M}$. For example $\kappa_{s}^{6,3}$ cannot be use to discriminate QPSK and 8-PSK; however, this can improve the capability of a feature vector to discriminate BPSK and 16-QAM.

In this work, every possible combination of feature vectors of lengths 1 to 7 with cumulant-based features up to the order of eight are investigated via simulations. Fig. 3 shows the classification rates for the best feature vectors with different lengths. The curves for feature vectors with a length between two and six are on top of each other. The performance difference between the classifier that uses the feature vector $\mathbf{K}=\left[\left|\kappa_{s}^{8,2}\right|,\left|\kappa_{s}^{8,4}\right|\right]^{T}$ and ALRT is only $4 \mathrm{~dB}$ at a $P_{c c}$ of 0.9 . Using the feature vector $\mathbf{K}=\left[\left|\kappa_{s}^{8,2}\right|,\left|\kappa_{s}^{8,4}\right|\right]^{T}$ instead of using only one cumulant as a feature leads to a gain of about $1.5 \mathrm{~dB}$ at a $P_{c c}$ of 0.9. The simulation results indicate that by using more than two cumulants does not improve the performance significantly.

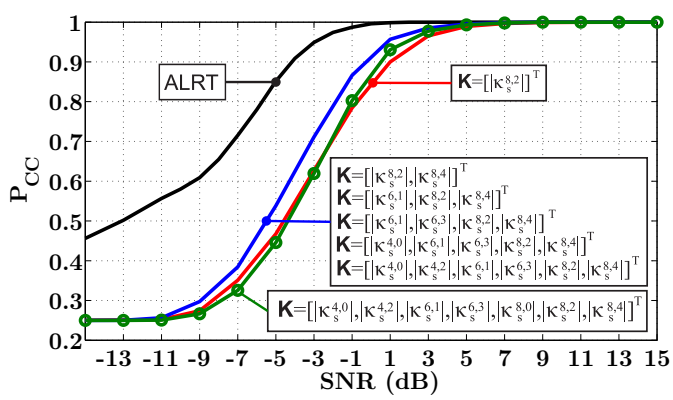

Fig. 3. Performance of the proposed algorithm with feature vectors with different lengths. The best feature combinations are plotted in case of CSI with $\mathrm{ZF}$ equalization.

The algorithm which uses the feature vector $\mathbf{K}=$ $\left[\left|\kappa_{s}^{8,2}\right|,\left|\kappa_{s}^{8,4}\right|\right]^{T}$ provides a good performance with relatively low computational complexity. In Fig. 4, the performance of this feature vector is investigated, when the transmitted symbol streams from each transmit antenna are separated using the JADE algorithm. At high SNR, the performance of JADE and perfect CSI with ZF equalization are similar, while at a lower SNR the former loses only about $0.25 \mathrm{~dB}$ when compared with the latter.

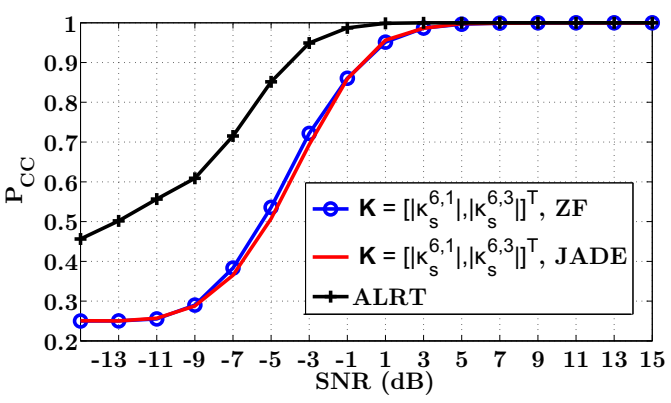

Fig. 4. Performance of the proposed algorithm with and without CSI, as well as the ALRT.

\section{CONCLUSiON}

A feature-based approach to automatic modulation classification (AMC) for multiple-input multiple-output (MIMO) signals is proposed, which exploits higher-order cumulants. The classification performance of feature vectors, which include diverse higher-order cumulants is intensively investigated via simulations. It is shown that an improved performance is achieved with eighth-order cumulants. Perfect channel state information (CSI) is considered, as well as the realistic case of blind channel estimation through the joint approximate diagonalization of eigen-matrices (JADE) algorithm. Results show that a reasonably close performance is obtained in the latter case, when compared with the former. The performance of the proposed algorithm is also compared with that of the optimal average likelihood ratio test (ALRT). While ALRT requires a priori information on the channel and suffers of high computational complexity, the proposed algorithm does not display such drawbacks. These results indicate the suitability of our proposed algorithm for practical implementation.

\section{REFERENCES}

[1] F. Jondral, "Automatic classification of high frequency signals," Signal Processing, vol. 9, pp. 177-190, 1985.

[2] A. Swami and B. Sadler, "Hierarchical digital modulation classification using cumulants," IEEE Transactions on Communications, vol. 48, no. 3 , pp. $416-429$, Mar. 2000.

[3] C. Spooner, "Classification of co-channel communication signals using cyclic cumulants," in Proc. IEEE Asilomar, 1995, pp. 531 -536.

[4] - "On the utility of sixth-order cyclic cumulants for rf signal classification," in Proc. IEEE Asilomar, 2001, pp. 890 -897.

[5] O. Dobre, Y. Bar-Ness, and W. Su, "Higher-order cyclic cumulants for high order modulation classification," in IEEE Milcom, 2003, pp. 112 117.

[6] O. Dobre, A. Abdi, Y. Bar-Ness, and W. Su, "Survey of automatic modulation classification techniques: classical approaches and new trends," IET Communications, vol. 1, no. 2, pp. 137 -156, April 2007.

[7] V. Choqueuse, S. Azou, K. Yao, L. Collin, and G. Burel, "Blind modulation recognition for mimo systems," MTA Review, vol. 19, pp. 183-196, 2009

[8] M. Mhlhaus, M. ner, O. Dobre, H. Jkel, and F. Jondral, "Automatic modulation classification for mimo systems using fourth-order cumulants," in Proc. IEEE VTC Fall, 2012.

[9] J. Cardoso and A. Souloumiac, "Blind beamforming for non-gaussian signals," IEE Proceedings on Radar and Signal Processing,, vol. 140, no. 6, pp. $362-370$, Dec. 1993.

[10] W. Gardner and C. Spooner, "The cumulant theory of cyclostationary time-series, part 1: Foundation," IEEE Transactions on Signal Processing, vol. 42, no. 12, pp. 3387 -3408, Dec. 1994. 